\title{
Few-layer-graphene with high yield and low sheet resistance via mild oxidation of natural graphite $\dagger$
}

Cite this: RSC Adv., 2017, 7, 35717

\author{
S. Kim, J. Y. Lee (D) and T. H. Yoon (D) *
}

The mild oxidation of natural graphite was obtained via the modified Hummers method by employing an oxidation temperature of $20{ }^{\circ} \mathrm{C}$ and $\mathrm{KMnO}_{4}$ loading of $2 \mathrm{~g}$, while the degree of mild oxidation was controlled by varying the oxidation time from 30 to 60,90 or $120 \mathrm{~min}$. The mild oxidation of graphite was demonstrated by X-ray diffraction (XRD), along with UV-vis spectroscopy, scanning electron microscopy (SEM) and X-ray photoelectron spectroscopy (XPS). Then, MOG (mildly-oxidized graphite) was grafted with aryldiazonium salts of sulfonic acid (ADS) to afford good water dispersion, followed by sonication exfoliation and centrifugation to isolate the few-layer-graphene (FLG). The FLG was characterized by transmission electron microscopy (TEM), atomic force microscopy (AFM) and Raman spectroscopy, and its sheet resistance $\left(R_{\mathrm{s}}\right)$, yield and water dispersion stability were also evaluated. The obtained FLG had low $R_{\mathrm{s}}\left(3 \times 10^{2}\right.$ to $\left.5.8 \times 10^{4} \Omega \square^{-1}\right)$, high yield (19-85\%), and good stability in water (79-86\% after 4 week aging).

Received 30th May 2017 Accepted 12th July 2017

DOI: $10.1039 / \mathrm{c} 7 \mathrm{ra0} 0042 \mathrm{~d}$

rsc.li/rsc-advances single layered graphene is not critical. In addition, the small size of graphene $(\sim 1 \mu \mathrm{m})$ resulting from physical exfoliation also poses a problem at times, ${ }^{\mathbf{1 2}}$ but not always.

Extensive studies have also been conducted on chemical exfoliation, which generates graphene oxide (GO) with high yield, good water dispersion and large size (similar to natural graphite). ${ }^{13}$ But this method has the critical drawback of providing graphene derivatives of poor properties due to damaged $\mathrm{sp}^{2}$ carbon bonds in the molecular structures, ${ }^{\mathbf{1 2}}$ which has led to considerable research on the reduction process aimed at restoring the bonds and recovering the original properties. ${ }^{15-18}$ Despite subsequent success in the effort, two critical concerns still remain: incomplete reduction ${ }^{\mathbf{1 9}}$ and deterioration of good water dispersion. ${ }^{20}$ The latter can be attributed to the loss of oxygen functional groups upon reduction $^{\mathbf{1 5}}$ and thus, it can be resolved by the grafting of functional moieties to the graphite oxide. ${ }^{21}$ The problem of incomplete reduction, however, is believed to arise from hole defects on the basal plane, which are formed by broken $\mathrm{sp}^{2}$ carbon bonds upon over-oxidation, ${ }^{\mathbf{1 4 , 2 2}}$ and may not be resolved by the reduction process. Thus, attempts were made to heal such defects with aromatic organic molecules, ${ }^{23}$ for example, but were not completely successful.

Complete reduction, however, can possibly be achieved only if graphite oxide has a low defect density ${ }^{24}$ without any hole defects, which can be obtained via partial or mild oxidation rather than full oxidation. In fact, there have been a number of studies on the mild oxidation of graphite by varying the oxidant loading, ${ }^{25-28}$ oxidation temperature ${ }^{29-33}$ and/or oxidation time ${ }^{34}$ in the Hummers or Brodie's method. In these studies, however, one critical concern was the demonstration of mild oxidation. 
Despite claims of mild oxidation, only about half of these studies provided clear supporting evidence. For the characterization, a number of techniques were used, including X-ray diffraction (XRD), X-ray photoelectron spectroscopy (XPS), Raman spectroscopy and thermogravimetric analyser (TGA). Of these, XRD appeared to be better suited for demonstrating mild oxidation since it can provide the characteristic peaks of graphite and graphite oxide. Thus, the observation of peaks for both graphite and graphite oxide would mean mild oxidation, while the appearance of only graphite oxide peak would indicate full oxidation. Based on such consideration, literature studies can be divided into three groups: (i) a group with both graphite and graphite oxide peaks, demonstrating mild oxidation, ${ }^{27,28,30,31,34}$ (ii) a group with only graphite oxide peak, indicating full oxidation, ${ }^{26,32,33}$ and (iii) a group with no XRD characterisation. ${ }^{25,29}$ Most of the studies in the first group employed one or two oxidation conditions for mild oxidation, with the exception of two studies which increased $\mathrm{KMnO}_{4}$ loading, ${ }^{27}$ or oxidation time, ${ }^{34}$ and demonstrated mild to full oxidation based on the XRD analysis. Unfortunately, neither properties nor yield were reported, even though a wide range of FLG properties and yields were expected, given the wide range of mild oxidation. In this study, therefore, mild oxidation was attempted via the modified Hummers method by employing a $\mathrm{KMnO}_{4}$ loading of $2 \mathrm{~g}$ and oxidation temperature of $20^{\circ} \mathrm{C}$. The degree of mild oxidation was controlled by varying the oxidation time, while the sheet resistance and yield of FLG were evaluated as a function of oxidation time. In addition, the water dispersion stability of FLG was also studied to demonstrate the effect of ADS grafting.

\section{Experimental}

\section{Materials}

Natural graphite (325 mesh, 99.8\%, metals basis) was purchased from Alfa Aesar (Ward Hill, MA, USA), and $\mathrm{KMnO}_{4}$ (ACS reagent, >99\%) was supplied by Sigma-Aldrich (St. Louis, MO, USA). $\mathrm{HCl}$ (extra pure, $>35 \%$ ), $\mathrm{H}_{2} \mathrm{SO}_{4}$ (extra pure, $>95 \%$ ) and $\mathrm{H}_{2} \mathrm{O}_{2}$ (extra pure, $>35 \%$ ) were purchased from OCI (Korea).

\section{Mild oxidation of natural graphite}

For mild oxidation in this study, the Hummers method was modified by lowering the $\mathrm{KMnO}_{4}$ loading from 3 to $2 \mathrm{~g}$ and the oxidation temperature from 35 to $20^{\circ} \mathrm{C}$, in addition to omitting the 2nd step oxidation at $98{ }^{\circ} \mathrm{C}$ (Fig. S1, ESI $\dagger$ ). The oxidation time was varied from 30 to 60,90 or $120 \mathrm{~min}$ to obtain controlled mild oxidation. Briefly, the oxidation was carried out by charging the fuming $\mathrm{H}_{2} \mathrm{SO}_{4}(46 \mathrm{~mL})$ and $2 \mathrm{~g}$ of natural graphite into a round-bottom flask in a $20{ }^{\circ} \mathrm{C}$ water bath. Once the graphite was completely soaked, it was slowly stirred to obtain a homogenous mixture. Next, $4 \mathrm{~g}$ of $\mathrm{KMnO}_{4}$ was slowly added ( $\sim 5 \mathrm{~min})$ under slow stirring, followed by oxidation reaction for $30,60,90$ or $120 \mathrm{~min}$, while maintaining the water bath at $20{ }^{\circ} \mathrm{C}$ throughout the oxidation process. However, $\mathrm{NaNO}_{3}$ was not added since it is not necessary for mild oxidation, but causes the problems reported previously. ${ }^{35}$ For convenience, the samples were named MOG-30, MOG-60, MOG90 or MOG-120 to indicate mildly-oxidized graphite (MOG) and oxidation time.

Upon completion of the oxidation, the reaction mixture was transferred into a $2 \mathrm{~L}$ glass bottle containing $1.8 \mathrm{~L}$ of DIW. After adding $20 \mathrm{~mL}$ of $\mathrm{H}_{2} \mathrm{O}_{2}$, the volume of the final solution was adjusted to $2 \mathrm{~L}$ by adding DIW. Next, the solution was transferred to a $2 \mathrm{~L}$ mess cylinder and subjected to aging overnight, after which the top solution ( 90\%) was decanted, and 10\% $\mathrm{HCl}$ solution was added again to adjust the volume to $2 \mathrm{~L}$. This process was repeated once using the $10 \% \mathrm{HCl}$ solution and then repeated twice using DIW. Finally, the concentration of MOG solution was adjusted to $4 \mathrm{mg} \mathrm{mL}{ }^{-1}$ for the grafting of aryl diazonium salts of sulfonic acid (ADS). Grafting was carried out on the whole solution in a single round, rather than dividing the solution into several batches, in order to save time and minimize experimental error.

\section{Grafting of aryldiazonium salts of sulfonic acid (ADS)}

The ADS grafting of MOG was carried out to enhance the water dispersion of FLG after exfoliation, since MOG may not have enough oxygen functional groups to provide good water dispersion due to mild oxidation. ADS was synthesized ${ }^{36}$ and grafted to MOG. ${ }^{37}$ Grafting was carried out by adding $100 \mathrm{~mL}$ of cold DIW $\left(\sim 5^{\circ} \mathrm{C}\right)$ in a $1 \mathrm{~L}$ round-bottom flask which was placed in an ice-water bath. When the temperature of DIW reached $5{ }^{\circ} \mathrm{C}, 1.6 \mathrm{~g}$ of ADS was added and stirred until it was completely dissolved. Next, $400 \mathrm{~mL}$ of the cold MOG solution $\left(4 \mathrm{mg} \mathrm{mL} \mathrm{mL}^{-1}\right)$ was added to the flask ( $1 \mathrm{mg}$ of ADS for $1 \mathrm{mg}$ of MOG) and reacted for $3 \mathrm{~h}$ in the ice-water bath $\left(\sim 5{ }^{\circ} \mathrm{C}\right)$. The remaining MOG solution was saved for characterization and other studies. At the end of the reaction, the concentration of the ADS-grafted MOG (MOG-ADS) was adjusted to $1 \mathrm{mg} \mathrm{mL}^{-1}$ by adding DIW. The solution was, then, centrifuged at $4000 \mathrm{rpm}$ for $1 \mathrm{~h}$ to remove the unreacted ADS, followed by neutralization with $\mathrm{NaOH}$ solution to $\sim \mathrm{pH} 7$.

\section{Sonication exfoliation of MOG}

The exfoliation of MOG-ADS was carried out using an ultrasonic cleaner (Hwasin Powersonic 605, $350 \mathrm{~W}$, Korea) at $40 \mathrm{kHz}$ for up to $36 \mathrm{~h}$. During the exfoliation, $4 \mathrm{~mL}$ of the aliquot was collected at set times and used for UV-vis absorption measurements at $750 \mathrm{~nm}$ to monitor the progress of exfoliation. The collected solutions (with $10 \times$ dilution) were centrifuged at $1000 \mathrm{rpm}$ for $2 \mathrm{~h}$ to estimate the FLG generation based on UV-vis absorption measurement. Upon completion of the exfoliation, the solution was centrifuged, and the supernatant containing FLG was purified via dialysis with a cellulose membrane (SpectrumLabs, $d=25.5 \mathrm{~mm}, \mathrm{MWCO}=6-8 \mathrm{kDa}$ ) until no peaks were observed in the 200-300 $\mathrm{nm}$ range of UV-vis absorption. Finally, the FLG was filtered and characterized.

\section{Characterization of MOG}

All solutions of MOG, MOG-ADS and FLG were analysed by UVvis spectroscopy (Agilent, 8453). The absorption was measured using a standard rectangular-shape quartz cuvette $(3.5 \mathrm{~mL})$ with 
diluted solution $(10 \times)$ and thus, reported absorbance was 10 times of observed values, but no other conversions were made. The MOG was also characterized by field-emission scanning electron microscopy (FE-SEM, Jeol, JSM-7500F) at $15 \mathrm{keV}$ with $\mathrm{Au}-\mathrm{Pd}$ coating, and also by XRD (Empyrean, X'Pert PRO Multi Purpose) with a Cu (1.8 kW) target. XPS (VG Multilab 2000) with a monochromatized $\mathrm{Al} \mathrm{K} \alpha$ source $(1486.6 \mathrm{eV})$ was utilized to characterize the MOG and MOG-ADS samples. Survey scans as well as narrow scans of $\mathrm{C}_{1 \mathrm{~s}}, \mathrm{O}_{1 \mathrm{~s}}$ and $\mathrm{S}_{2 \mathrm{p}}$ peaks were obtained, while the $\mathrm{C}_{1 \mathrm{~s}}$ peak was deconvoluted by using the XPSPEAK software (The Chinese University of Hong Kong). The FLG was characterized by TEM (Jeol-2100) using samples on a carbon coated copper grid. It was also analysed by AFM (XE-100, Park Systems, Korea) in tapping mode and Raman spectroscopy (UniRam-5000, UniThink, Korea) equipped with an $532 \mathrm{~nm}$ laser. The samples for these analyses were prepared by drop coating on a Si wafer. On the other hand, the properties of FLG were evaluated by measuring the sheet resistance via the 4-point probe method (CMT-SR2000, Changmin Tech., Korea) with thin films having a diameter of $1.8 \mathrm{~cm}$ and weight of $\sim 3 \mathrm{mg}$. At least 3 samples were measured and the results were averaged. The yield of FLG was calculated by filtering and measuring the weight of the FLG from supernatant and the pellets upon centrifugation. Stability of the FLG solution was also evaluated by measuring their UV-vis absorption after aging for $24 \mathrm{~h}$ to 4 weeks. For comparison, the stability of MOG and MOG-ADS solutions was also studied.

\section{Results and discussion}

\section{Mild oxidation of natural graphite}

The mild oxidation of natural graphite was attempted via the modified Hummers method under the following conditions: oxidation temperature of $20{ }^{\circ} \mathrm{C}, \mathrm{KMnO}_{4}$ loading of $2 \mathrm{~g}$, and oxidation time ranging from 30 to 60,90 or $120 \mathrm{~min}$ to control the degree of mild oxidation. At the end of oxidation, all solutions were black in colour, demonstrating mild oxidation, based on a comparison with black obtained from mild oxidation ${ }^{24}$ and light brown from the Hummers method..$^{25,27}$ The MOG solutions provided rapidly increased and then nearly flat UV-vis absorption spectra (Fig. 1). These are similar to the almost flat spectra from the graphene solution via sonication exfoliation of graphite in $N$-methylpyrrolidine (NMP) ${ }^{6}$ and reduced graphene oxide. $^{39}$ The UV-vis absorption spectra in this study clearly demonstrated mild oxidation. As noted, the UV-vis absorption of MOG solutions at $750 \mathrm{~nm}$ increased with oxidation time (Fig. 1), providing 7.2, 9.3, 10.4 and 10.7 for MOG-30, MOG-60, MOG-90 and MOG-120, respectively, which can be attributed to the increased oxidation exfoliation with oxidation time. It was also noted that the UV-vis absorption decreased slowly at above $600 \mathrm{~nm}$, possibly due to higher degree of oxidation.

\section{Characterization of MOG}

The SEM characterization of MOG showed an expanded structure for MOG-30 (Fig. 2a), but a platelet structure for MOG-120 (Fig. 2d), while mixture of these two structures for MOG-60 and

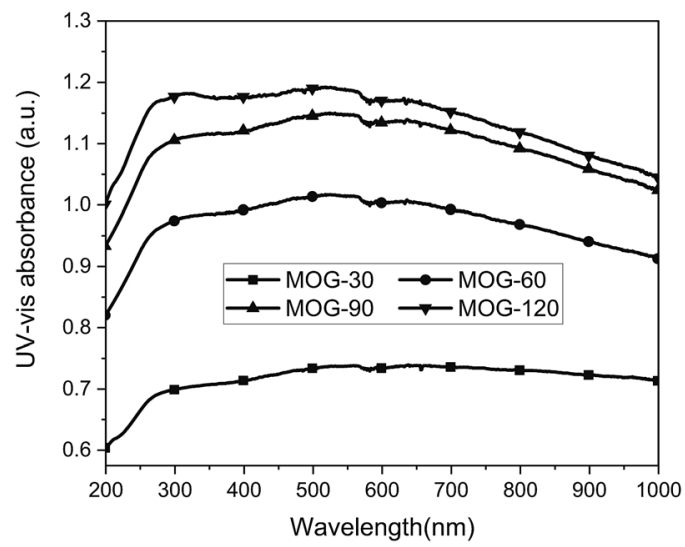

Fig. 1 UV-vis absorbance of MOG solutions prepared with different reaction time.

MOG-90. The former is similar to the expanded structure of graphite oxide from a previous study, ${ }^{\mathbf{4 0}}$ suggesting a low degree of exfoliation due to short oxidation time in combination with mild oxidation condition in this study. However, a longer oxidation time would mean an increased degree of exfoliation, leading to a platelet structure, like the one in MOG-120 (Fig. 2d). The increased UV-vis absorption with oxidation time supports this hypothesis (Fig. 1). One can see that the platelet MOG in this study had a thickness of several microns, which is still much thicker than the graphene $(\sim 0.34 \mathrm{~nm})$, again owing to mild oxidation. SEM micrographs reveal that the size of the platelet MOG was $\sim 20 \mu \mathrm{m}$ after oxidation, which is similar to the average size of as-received graphite particles (325 mesh), indicating that no appreciable fragmentation occurred during oxidation.

XRD was utilized to demonstrate the mild oxidation of MOG (Fig. 3). All samples exhibited three major peaks at 26.5, 13.5 and $8.5^{\circ}$, with the exception of MOG-30 which showed a peak at 16.5 instead of $13.5^{\circ}$. Considering the peak position and its intensity, the peak at $26.5^{\circ}$ can be assigned to graphite, ${ }^{26}$ while the one peak at $13.5^{\circ}$ can be attributed to the mildly-oxidized graphite. ${ }^{27,29,31,34}$ On the other hand, the peak at $16.5^{\circ}$ in MOG30 can be attributed to the intermediate compounds, ${ }^{30}$ resulting from a short oxidation time of $30 \mathrm{~min}$ since it appeared with only MOG-30. In comparison, the peak at $8.5^{\circ}$, which was observed in all samples, was seldom reported in the literature unless an intercalation agent was used..$^{\mathbf{6 , 4 1}}$ It can be seen that the peak intensity gradually decreased with oxidation time, indicating that it is not from graphite oxide. Given that the peak is not observed in pristine graphite, one can surmise that this peak is from another intermediate compound that is converted to MOG, appearing the peak at $13.5^{\circ}$ and then increasing its intensity as the oxidation time increased. Furthermore, considering the peak intensity decrease at $8.5^{\circ}$, this peak can be correlated to the MOG with an expanded structure resulting from mild oxidation (as shown in SEM images in Fig. 2a), since this structure decreased in both number and thickness as the oxidation time increased. Given the large apparent interlayer spacing observed in the SEM (1-2 $\mu \mathrm{m})$, the interlayer spacing of 

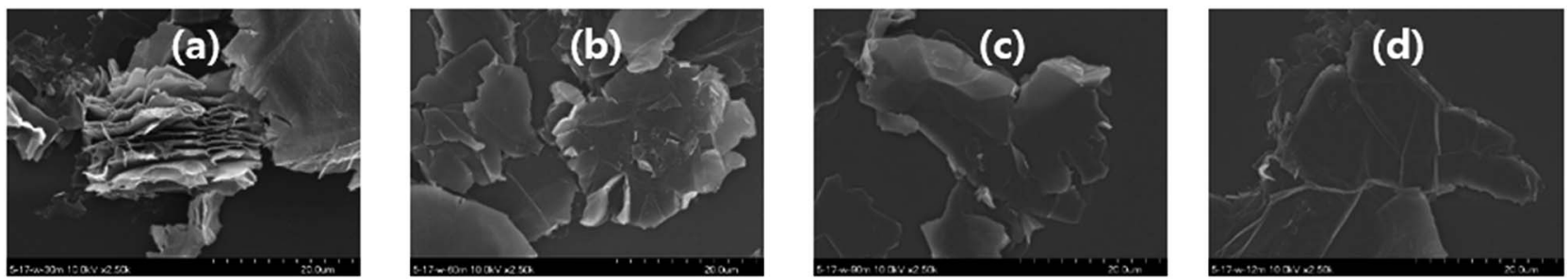

Fig. 2 SEM of mildly-oxidized graphite with (a) MOG-30, (b) MOG-60, (c) MOG-90, and (d) MOG-120.

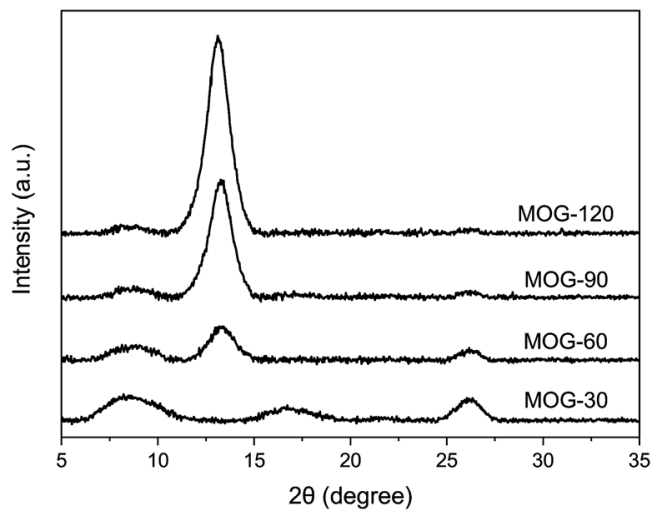

Fig. 3 XRD analysis of mildly oxidized graphite.

$10.4 \AA$ Abtained from the calculation $\left(8.5^{\circ}\right)$ can be attributed to the diffraction occurring deep inside the expanded structure. It should be noted that the presence of peaks at 26.5 and $13.5^{\circ}$ strongly support the case of mild oxidation in this study, while the peaks at 8.5 and $16.5^{\circ}$ serve as evidence for low-mild oxidation.

The mild oxidation of MOG was also demonstrated by the XPS analysis, as shown in Fig. 4. All samples showed strong C$\mathrm{C}$, weak $\mathrm{C}-\mathrm{O}$ and much weaker $\mathrm{C}=\mathrm{O}$ peaks at $284.6,286.5$ and $288.8 \mathrm{eV}$, respectively, similar to the mild oxidation spectra in the literature. ${ }^{32}$ The intensity of $\mathrm{C}-\mathrm{O}$ and $\mathrm{C}=\mathrm{O}$ peaks increased with oxidation time, resulting in a decrease of $\mathrm{C} / \mathrm{O}$ atomic ratio from 4.1 (MOG-30) to 3.6 (MOG-60), 3.1 (MOG-90) and 2.8 (MOG-120) (Table S1, ESI $\dagger$ ). Based on the comparison of these results with 2.04 or 2.17-2.6 obtained from fully-oxidized and mildly-oxidized graphite, ${ }^{42}$ respectively, as well as with 12.5 from the reduced-graphite oxide, ${ }^{43}$ it can be said that mild oxidation was successfully obtained in this study, along with a well-controlled degree of mild oxidation.
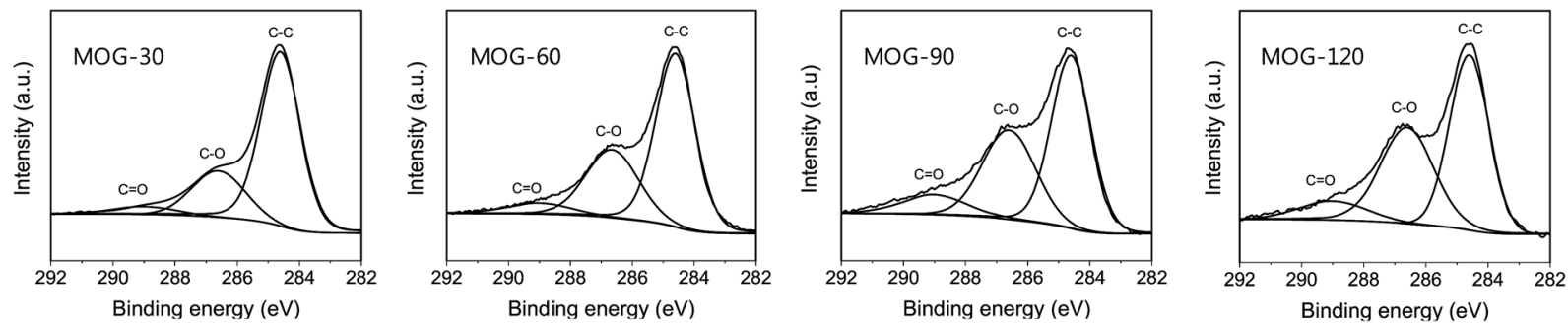

Fig. 4 Deconvolution of $C_{1 s}$ peak from MOG after XPS analysis.

\section{ADS grafting of MOG}

The grafting of ADS to enhance the water dispersion of FLG was confirmed by the XPS analysis, which showed a clear sulphur $\left(\mathrm{S}_{2 \mathrm{p}}\right)$ peak at $\sim 168 \mathrm{eV}$ (ref. 44) (Fig. S2, ESI $\dagger$ ) with $2.2-1.5 \%$ atomic concentration (Table S1, ESI $\dagger$ ). This can be compared with $\sim 0.7 \%$ sulphur obtained from MOG (without ADS grafting) in Table S1, ESI, $\dagger$ which is likely from the sulfuric acid used for oxidation. ${ }^{45}$ The grafting of ADS was also supported by slightly weaker intensities of $\mathrm{C}-\mathrm{O}$ and $\mathrm{C}=\mathrm{O}$ peaks than those from the corresponding MOG without ADS grafting (Fig. S3, ESI $\dagger$ ), which in turn resulted in a slightly higher atomic concentration of carbon in MOG-ADS, compared to MOG, thus, higher C/O ratios (Table S1, ESI $\dagger$ ). This can be attributed to the extra C-C moieties from the ADS. The grafting of ADS was also demonstrated by the better stability of MOG-ADS than that of MOG, which will be discussed later.

\section{Exfoliation of MOG via sonication}

The exfoliation of MOG-ADS was carried out by sonication and monitored by UV-vis absorption measurements (Fig. 5a). The absorption of MOG-ADS increased sharply with $1 \mathrm{~h}$ exfoliation, resulting in 143, 187, 156 and 146\% increase for MOG-ADS-30, MOG-ADS-60, MOG-ADS-90 and MOG-ADS-120, respectively (Table S2, ESI $\dagger$ ). These sharp increases can be explained by low UV-vis absorption from as-oxidized MOG solutions due to mild oxidation and thus expanded structure, which can be easily exfoliated via sonication. This can be compared with slow increase in UV-vis absorption via sonication exfoliation of pristine graphite, being attributed to no oxidation and thus no expanded structure, as reported previously. ${ }^{38}$

The highest UV-vis absorption was obtained from MOGADS-60, followed by MOG-ADS-90, MOG-ADS-120 and MOGADS-30, which is different from the order of as-oxidized MOG solutions. This can be explained by two factors: the exfoliation 

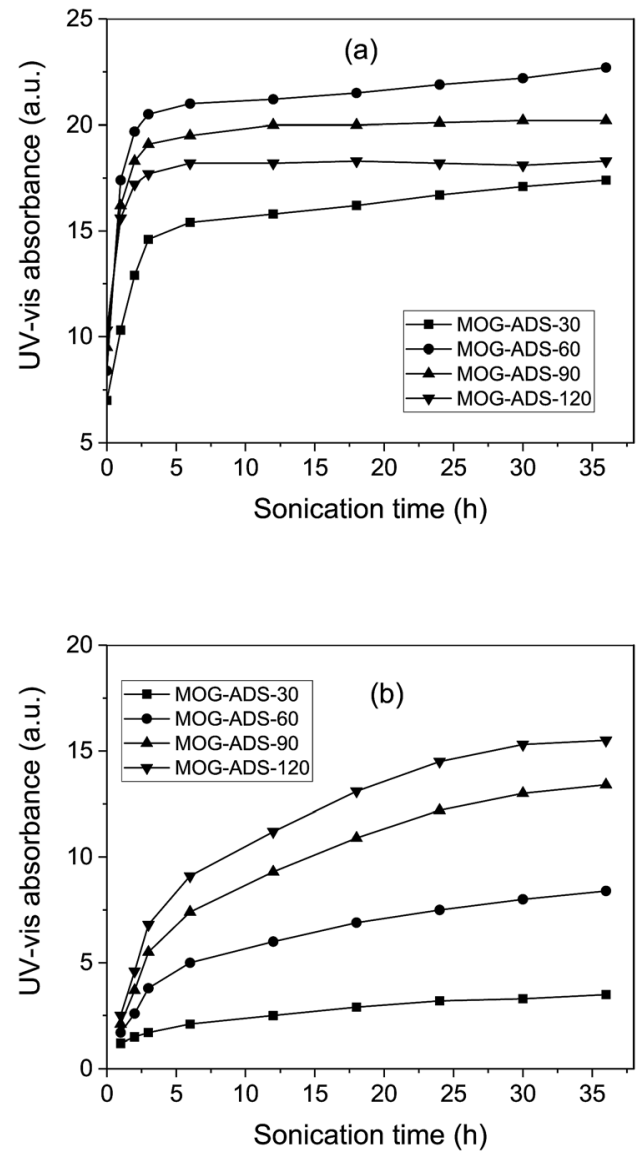

Fig. 5 UV-vis absorbance of MOG solutions upon sonication exfoliation (a) and subsequent centrifugation (b).

of MOG and damage to the $\mathrm{sp}^{2}$ carbon bonds. Both increase with oxidation time, but the former leads to increased absorption and the latter to decreased absorption. In this sense, the lower UV-vis absorption of MOG-ADS-30 can be explained by slow exfoliation, despite low damage to the $\mathrm{sp}^{2}$ carbon bonds. On the other hand, the lower absorption of MOG-ADS-90 and MOG-ADS-120 can be attributed to high damage to the $\mathrm{sp}^{2}$ carbon bonds, despite fast exfoliation. In other words, MOGADS-60 had an optimum degree of mild oxidation, providing fairly fast exfoliation, but fairly low damage to the $\mathrm{sp}^{2}$ carbon bonds.

With further sonication, the absorption increased rather rapidly at $2 \mathrm{~h}$, followed by a slow increase or near saturation (Fig. 5a). At $36 \mathrm{~h}$ of sonication, MOG-ADS-60 still showed the highest absorption, followed by MOG-ADS-90, MOG-ADS-120 and MOG-ADS-30. This can again be explained by the increased exfoliation and increased damage to the $\mathrm{sp}^{2}$ carbon bonds with longer oxidation time. However, the largest increase was obtained from MOG-ADS-30, followed by MOG-ADS-60, MOG-ADS-90 and MOG-ADS-120, correlating to the degree of oxidation; that is to say, lower the degree of oxidation, larger the room for exfoliation.

Unfortunately, these UV-vis absorptions cannot be directly correlated to FLG generation, and thus, the solutions were centrifuged, followed by UV-vis absorption measurements to estimate FLG generation. Of course, the weight measurement was also carried out for the yield calculation, which will be discussed later. Upon centrifugation, the absorption increased with sonication time slowly (MOG-ADS-30), moderately (MOGADS-60), or rather rapidly (MOG-ADS-90, MOG-ADS-120), as shown in Fig. 5b. In addition, the highest UV-vis absorption was observed from MOG-ADS-120, followed by MOG-ADS-90, MOGADS-60 and MOG-ADS-30, which is similar to the results of asoxidized solutions, but different from that of as-sonicated (Fig. 5a). This trend can be explained by the easier fragmentation of MOG-ADS flakes upon sonication with longer oxidation time. The fragmentation was supported by the TEM analysis in this study (will be discussed later), as well as by previous studies. ${ }^{39,46}$ In other words, the longer the oxidation time or the sonication time, the thinner the FLG generated, and thus, easier the fragmentation. Upon centrifugation, the supernatant of MOG-ADS-30, MOG-ADS-60, MOG-ADS-90 and MOG-ADS-120 provided UV-vis absorptions of 3.4, 8.4, 13.4 and 15.5, respectively, which correspond to $20,59,75$ and $89 \%$ of the asexfoliated solutions (Table S2, ESI $\dagger$ ). These can be used as a rough estimation of the FLG yield, while the exact yield is calculated from the weight measurements.

\section{Characterization of FLG from mild oxidation}

The TEM characterization of FLG showed a highly transparent FLG having a size as large as $1 \mu \mathrm{m}$, but 11 FLG flakes of MOG-60 had an average size of $0.52 \mu \mathrm{m}$ (Fig. 6a). Similar results were also observed in all other samples in the study (Fig. S4, ESI $\dagger$ ), which are comparable to $\sim 0.5 \mu \mathrm{m}$ observed from the sonication exfoliation of natural graphite without oxidation. ${ }^{7}$ Considering the size of the platelet MOG (325 mesh), the small size can be attributed to severe fragmentation resulting from sonication, as suggested by the increased UV-vis absorption upon centrifugation (Fig. 5b). Thus, it may be necessary to decrease the sonication time in order to increase the size of FLG.

The AFM analysis of FLG resulted in an average size of $\sim 0.56$ $\mu \mathrm{m}$ from four FLG flakes (Fig. 6b), but as large as $1 \mu \mathrm{m}$ size was also observed, similar to the results of TEM studies. Of course, a similar behaviour was observed in other samples (Fig. S5, ESI $\dagger$ ), as well. The thickness of FLG, however, was $2-2.5 \mathrm{~nm}$ for all samples, exhibiting 3-4 layers (Fig. 6c) based on the interlayer space of fully-oxidized graphite oxide. ${ }^{25,34}$ The results of Raman spectroscopy on FLG are shown in Fig. 6d. A strong and broad D peak $\left(\sim 1340 \mathrm{~cm}^{-1}\right)$ and G peak $\left(\sim 1580 \mathrm{~cm}^{-1}\right)$ along with a very weak and broad $2 \mathrm{D}$ peak $\left(\sim 2700 \mathrm{~cm}^{-1}\right)$ were observed, being similar to that of graphite oxide with $\sim 3 \%$ defect on basal plane. ${ }^{24}$ These are similar to the spectra from mildly-oxidized graphite as previously reported, ${ }^{25,28,31}$ demonstrating again mild oxidation in this study.

\section{Property measurements of FLG after controlled mild oxidation}

The sheet resistance $\left(R_{\mathrm{S}}\right)$ of FLG, which was measured using a thin disc type FLG film ( $3 \mathrm{mg}$ ), was $3 \pm 0.5 \times 10^{2}, 4.6 \pm 0.5 \times$ $10^{3}, 1.6 \pm 0.2 \times 10^{4}$ and $5.8 \pm 0.5 \times 10^{4} \Omega \square^{-1}$ for MOG-ADS30, MOG-ADS-60, MOG-ADS-90 and MOG-ADS-120, 
(a)

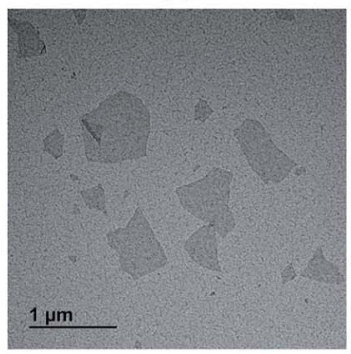

(b)

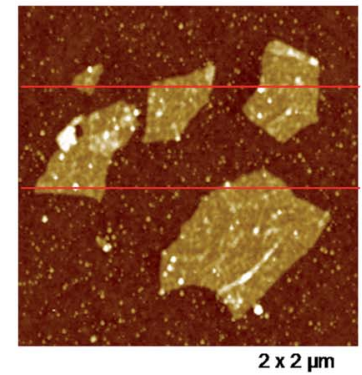

(c)
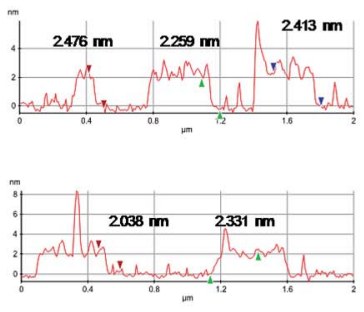

(d)

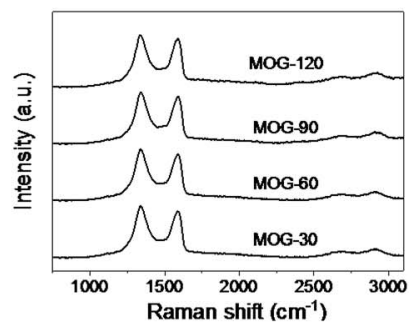

Fig. 6 Characterization of FLG from MOG by TEM (a), AFM (b, c) and Raman spectroscopy (d).

respectively (Table 1). A comparison of these values with those from CVD graphene $\left(\sim 1 \times 10^{2} \Omega \square^{-1}\right)$, graphene via sonication exfoliation in polar solvents $\left(\sim 1 \times 10^{3} \Omega \square^{-1}\right)$, or reduced graphene oxide ${ }^{1}\left(10^{3}\right.$ to $\left.10^{4} \Omega \square^{-1}\right)$ indicate that mild oxidation was realized in this study. As expected, the $R_{\mathrm{S}}$ increased with oxidation time, likely due to the increased damage to the $\mathrm{sp}^{2}$ carbon bonds, and correlated well with the results from UV-vis, XPS, XRD and Raman analysis. Moreover, the grafting of ADS may have had some effects on the sheet resistance, but was not investigated in this study.

The FLG yield obtained from the weight measurement provided 19, 55, 73 and 85\% for MOG-ADS-30, MOG-ADS-60, MOG-ADS-90 and MOG-ADS-120, respectively (Table 1) and can be compared with $20,59,75$ and $89 \%$ obtained from UV-vis absorption analysis (Table S2, ESI†). As expected, the yield increased with oxidation time as a result of the faster exfoliation of MOG, while unexpectedly high yields may be partially due to the ADS grafting, which enhances the water dispersion of FLG (will be discussed below). It is also interesting that the total weight of the supernatant and the precipitate is $\sim 2.0 \mathrm{mg} \mathrm{mL}^{-1}$ (Table S3, ESI $\dagger$ ), which is approximately double the weight of the graphite used for oxidation $\left(1 \mathrm{mg} \mathrm{mL} \mathrm{m}^{-1}\right)$. The weight gain may be attributed to ADS grafting, as well as oxidation, the latter which introduced oxygen functional groups,$^{29}$ was supported by the weight loss in the TGA analysis. ${ }^{20,32}$

Finally, the water dispersion of FLG was evaluated by measuring the UV-vis absorption of the FLG solutions upon 4 weeks of aging to demonstrate the effect of ADS grafting on the stability because the stability of graphene derivatives is critical for practical applications. ${ }^{47}$ As shown in Table 1, FLG solutions (MOG-ADS-sonication (36 h)-centrifugation (1k rpm/2 h))

Table 1 Sheet resistance, yield and stability of FLG from mildlyoxidized graphite

\begin{tabular}{lllll}
\hline & MOG-30 & MOG-60 & MOG-90 & MOG-120 \\
\hline$R_{\mathrm{S}}\left(\Omega \square^{-1}\right)$ & $3 \pm 0.5$ & $4.6 \pm 0.4$ & $1.6 \pm 2$ & $5.8 \pm 5$ \\
& $\times 10^{2}$ & $\times 10^{3}$ & $\times 10^{4}$ & $\times 10^{4}$ \\
FLG yield (\%) & 19 & 55 & 73 & 85 \\
Stability (\%) $^{a}$ & 79 & 85 & 86 & 86
\end{tabular}

${ }^{a}$ Based on absorbance of the solution after $36 \mathrm{~h}$ aging. showed the remaining UV-vis absorption of 79 (MOG-30), 85 (MOG-60) and 86\% (MOG-90 and MOG-120) after 4 weeks of aging, indicating excellent stability of the FLG, possibly owing to ADS grafting. Unfortunately, it was not possible to prepare a FLG solution from MOG without ADS grafting due to the highly hydrophobic nature of FLG, especially after sonication. Thus, the effect of ADS grafting was studied by comparing the stability of MOG and MOG-ADS solutions (without sonication) upon $24 \mathrm{~h}$ aging (Table S4, ESI $\dagger$ ). It can be seen that the ADS grafting provided much better stability (28-37\%), compared to the MOG solution (4-8\%). Similar stability was observed in all samples with ADS grafting, regardless of the oxidation time, possibly owing to the mild oxidation of all samples.

\section{Conclusions}

Mild oxidation was successfully realized via the modified Hummers method by employing an oxidation temperature of $20{ }^{\circ} \mathrm{C}$ and $\mathrm{KMnO}_{4}$ loading of $2 \mathrm{~g}$. The degree of mild oxidation was controlled by varying the oxidation time from 30 to 60,90 or $120 \mathrm{~min}$. Mild oxidation was clearly confirmed by XRD and supported by XPS, SEM and UV-vis spectroscopy, while TEM, AFM and Raman spectroscopy also demonstrated the successful preparation of FLG. The sheet resistance of FLG increased slowly with oxidation time and was $3 \pm 0.5 \times 10^{2}, 4.6 \pm 0.5 \times$ $10^{3}, 1.6 \pm 0.2 \times 10^{4}$ and $5.8 \pm 0.5 \times 10^{4} \Omega \square^{-1}$ for MOG-ADS30, MOG-ADS-60, MOG-ADS-90 and MOG-ADS-120, respectively. These are similar to the results of CVD graphene or reduced GO, indicating again mild oxidation and thus good electrical property of FLG. The yield of FLG also increased with oxidation time, resulting in a very high yield (as high as $85 \%$ ) for MOG-ADS-120. This can be attributed to the excellent stability of the FLG solution due to ADS grafting, which was demonstrated by the much better stability of the MOG-ADS solution, compared to the MOG solution.

\section{Acknowledgements}

This research was financially supported by the Ministry of Knowledge Economy (MKE), Korea Institute for Advancement of Technology (KIAT) through the Inter-ER Cooperation Projects (R00000499) and also by Gwangju Institute of Science and Technology/Program for Integrated Molecular System. 


\section{Notes and references}

1 Y. Zhu, S. Murali, W. Cai, Z. Li, J. W. Suk, J. R. Potts and

R. S. Rouff, Adv. Mater., 2010, 22, 3906.

2 R. K. Joshi, S. Alwarappan, M. Yoshimura, V. Sahajwalla and Y. Nishina, Appl. Mater. Today, 2015, 1, 1.

3 W. Ren and H. M. Cheng, Nat. Nanotechnol., 2014, 9, 726.

4 C. K. Chua, Z. Sofer and M. Pumera, Chem.-Eur. J, 2012, 18, 13453.

5 K. S. Novoselov, V. I. Fal'sko, L. Colombo, P. R. Gellert, M. G. Schwab and K. K. Kim, Nature, 2012, 490, 192.

6 Y. Hernandez, V. Nicolosi, M. Lotya, F. M. Blighe, Z. Sun, S. De, I. T. McGovern, B. Holland, M. Byrne, Y. K. Gun'Ko, J. J. Boland, P. Niraj, G. Duesberg, S. Krishnamurthy, R. Goodhue, J. Hutchison, V. Scardaci, A. C. Ferrari and J. N. Coleman, Nat. Nanotechnol., 2008, 3, 563.

7 M. Lotya, P. J. King, U. Khan, S. De and J. N. Coleman, ACS Nano, 2010, 4, 3155.

8 M. Vittori Antisari, A. Montone, N. Jovic, E. Piscopiello,

C. Alvani and L. Pilloni, Scr. Mater., 2006, 55, 1047.

9 K. R. Paton, et al., Nat. Mater., 2014, 13, 624.

10 M. Yi and Z. Shen, J. Mater. Chem. A, 2015, 3, 11700.

11 I.-Y. Jeon, Y. R. Shin, G. J. Sohn, H. J. Choi, S. Y. Bae, J. Mahmood, S. M Jung, J. M. Seo, M. J. Kim, D. W. Chang, L. Dai and J. B. Baek, Proc. Natl. Acad. Sci.,, 2012, 109, 5588. 12 Y. Zhu, W. Cai, R. D. Piner, A. Velamakanni and R. S. Ruoff, Appl. Phys. Lett., 2009, 95, 103104.

13 R. K. Singh, R. Kumar and D. P. Singh, RSC Adv., 2016, 6, 64993.

14 C. Gomez-Navarro, J. C. Meyer, R. S. Sundaram, A. Chuvilin, S. Kurasch, M. Burghard, K. Kern and U. Kaiser, Nano Lett., 2010, 10, 1144.

15 C. K. Chua and M. Pumera, Chem. Soc. Rev., 2014, 43, 291.

16 O. Jankovsky, P. Marvan, M. Novacek, J. Luxa, V. Mazanek,

K. Klimova, D. Sedmidubsky and Z. Sofer, Appl. Mater. Today, 2016, 4, 45.

17 S. Pei and H. M. Cheng, Carbon, 2012, 50, 3210.

18 S. Thakur and N. Karak, Carbon, 2015, 94, 224.

19 G. Eda, Y. Y. Lin, C. Mattevi, H. Yamaguch, H. A. Chen, I. S. Chen, C. W. Chen and M. Chhowalla, Adv. Mater., 2010, 22, 505.

20 M. Fang, K. Wang, H. Lu, Y. Yang and S. Nutt, J. Mater. Chem., 2010, 20, 1982.

21 T. Kuila, S. Bose, A. K. Mishra, P. Khanra, N. H. Kim and J. H. Lee, Prog. Mater. Sci., 2012, 57, 1061.

22 K. Erickson, R. Erni, Z. Lee, N. Alem, W. Gannett and A. Zettl, Adv. Mater., 2010, 22, 4467.

23 M. Zhang, R. R. Parajuli, D. Mastrogiovanni, B. Dai, P. Lo, W. Cheung, R. Brukh, P. L. Chiu, T. Zhou, Z. Liu, E. Garfunkel and H. He, Small, 2010, 10, 1100.

24 S. Eigler and A. Hirsch, Angew. Chem., Int. Ed, 2014, 53, 7720. 25 W. Gao, Q. Zheng, Z. Shen, H. Wu, Y. Ma, W. Guan, S. Wu, Y. Yu and K. Ding, Analyst, 2016, 141, 4713.
26 L. Wei, F. Wu, D. Shi, C. Hu, X. Li, W. Yuan, J. Wang, J. Zhao, H. Geng, H. Wei, Y. Wang, N. Hu and Y. Zhang, Sci. Rep., 2013, 3, 2636.

27 Y. Hu, S. Song and A. Lopez-Valdivieso, J. Colloid Interface Sci., 2015, 450, 68.

28 Y. Xu, K. Sheng, C. Li and G. Shi, J. Mater. Chem., 2011, 21, 7376.

29 S. Eigler, M. Enzelberger-Heim, S. Grimm, P. Hofmann, W. Kroener, A. Geworski, C. Dotzer, M. Roeckert, J. Xiao, C. Papp, O. Lytken, H.-P. Steinrueck, P. Mueller and A. Hirsch, Adv. Mater., 2013, 25, 3583.

30 Y. Hu, Z. Li, H. Li, Y. Liu and S. Song, RSC Adv., 2016, 6, 14859.

31 G. Eda, J. Ball, C. Mattevi, M. Acik, L. Artiglia, G. Granozzi, Y. Chabl, T. D. Anthopoulos and M. Chhowalla, J. Mater. Chem., 2011, 21, 11217.

32 G. Shao, Y. Lu, F. Wu, C. Yang, F. Zeng and Q. Wu, J. Mater. Sci., 2012, 47, 4400.

33 R. Kumar, S. Naqvi, N. Gupta, K. Gaurav, S. Khan, P. Kumar, A. Rana, R. K. Singh, R. Bharadwaj and S. Chand, RSC Adv., 2015, 5, 35983.

34 H. K. Jeong, M. H. Jin, K. P. So, S. C. Lim and Y. H. Lee, J. Phys. D: Appl. Phys., 2009, 42, 065418.

35 D. C. Marcano, D. V. Kosynkin, J. M. Berlin, A. Sinitskii, Z. Sun, A. Slesarev, L. B. Alemany, W. Lu and J. M. Tour, ACS Nano, 2010, 4, 4806.

36 X. Wang, R. Liu, M. M. Waje, Z. Chen, Y. Yan, K. Bozhilov and P. Feng, Chem. Mater., 2007, 19, 2395.

37 Y. Xu, Y. Wang, J. Liang, Y. Huang, Y. Ma, X. Wan and Y. Chen, Nano Res., 2009, 2, 343.

38 U. Khan, A. O'Neill, M. Lotya, S. De and J. N. Coleman, Small, 2010, 6, 864.

39 S. Yang, W. Yue, D. Huang, C. Chen, H. Lin and X. Yang, RSC $A d v ., 2012,2,8827$.

40 S. Park, J. An, J. R. Potts, A. Velamakanni, S. Murali and R. S. Ruoff, Carbon, 2011, 49, 3019.

41 C. Zeng, Z. Tang, B. Guo and L. Zhang, Phys. Chem. Chem. Phys., 2012, 14, 9838.

42 T. Szaboe, O. Berkesi, P. Forgo, L. Josepovits, Y. Sanakis, D. Petridis and I. Denkany, Chem. Mater., 2006, 18, 2740.

43 C. Mattevi, G. Eda, S. Agnoli, S. Miller, K. A. Mkhoyan, O. Celik, D. Mastrogiovanni, G. Granozzi, E. Garfunkel and M. Chhpwalla, Adv. Funct. Mater., 2009, 19, 2577.

44 N. Oger, Y. F. Lin, C. Labrugere, E. Le Grognec, F. Rataboul and F. X. Felpin, Carbon, 2016, 96, 342.

45 T. K. Mahto, R. Jain, S. Chandra, D. Toy, V. Mahto and S. K. Sahu, J. Environ. Chem. Eng., 2016, 4, 2933.

46 E. Y. Polyakova, K. T. Rim, D. Eom, K. Douglass, R. L. Oplia, T. F. Heinz, A. V. Teplyakov and G. W. Flynn, ACS Nano, 2011, 5, 6102.

47 C. K. Chua and M. Pumera, Small, 2015, 11, 1266. 